\title{
ESTABILIDADE DA PRODUÇÃO FORRAGEIRA EM CLONES DE CAPIM-ELEFANTE (Pennisetum purpureum Schum.) ${ }^{1}$
}

\author{
ROGÉRIO FIGUEIREDO DAHER ${ }^{2}$ \\ MESSIAS GONZAGA PEREIRA ${ }^{3}$ \\ ANTÔNIO TEIXEIRA DO AMARAL JÚNIOR ${ }^{3}$ \\ ANTÔNIO VANDER PEREIRA ${ }^{4}$ \\ FRANCISCO JOSÉ DA SILVA LÉDO ${ }^{4}$ \\ MÁSKIO DAROS ${ }^{2}$
}

\begin{abstract}
RESUMO - Quatorze clones e três cultivares de capimelefante foram avaliados no delineamento em blocos ao acaso, com quatro repetições. Objetivou-se estimar parâmetros de estabilidade pelas metodologias de Plaisted \& Peterson (1959), Lin \& Binns (1988) e Kang (1988), com base na produção de matéria seca (MS), em
\end{abstract}

$\mathrm{kg} / \mathrm{ha}$.corte, num total de 12 cortes (medidas repetidas no tempo como ambientes). O clone CNPGL 91 F11-2 e a cultivar Pioneiro apresentaram alta estabilidade e produtividade pelos métodos utilizados, destacando-se como promissores para as condições de Campos dos Goytacazes, RJ.

TERMOS PARA INDEXAÇÃO: Capim-elefante, clones, estabilidade, recomendação de cultivares, produção de matéria seca, Pennisetum.

\section{STABILITY OF FORAGE PRODUCTION IN CLONES OF ELEPHANTGRASS (Pennisetun purpureum Schum.)}

\begin{abstract}
Fourteen clones and three cultivars of elephant grass were evaluated in a randomized block experimental design with four repetitions. The objective of this study was to estimate stability parameters related to dry matter (DM) production in $\mathrm{kg} / \mathrm{ha}$.cut, based in methodologies of Plaisted \& Peterson (1959), Lin \&
\end{abstract}

Binns (1988) and Kang (1988), resulted of twelve cuts (as environments). The clone CNPGL 91 F11-2 and Pioneiro cultivar presented high stability and production based in these methodologies, consisting in satisfactory genetic materials adapted to Campos dos Goytacazes, RJ conditions.

INDEX TERMS: Clones, crops recommendation, dry matter production, elephantgrass, stability, Pennisetum.

\section{INTRODUÇÃO}

As capineiras constituem grande reserva de forragem para a época mais crítica do ano (época da seca), sendo o capim-elefante a forrageira mais utilizada, devido ao seu elevado potencial para produção de forragem de boa qualidade (EMBRAPA, 1993). Inúmeras cultivares e híbridos foram avaliados no Brasil em vários locais (Mozzer, 1986; Daher et al., 1990). Com o desenvolvimento de novos clones híbridos originados do programa de melhoramento da espécie na Embrapa Gado de Leite, tornam-se necessárias a avaliação e sele- ção desses materiais nos locais onde serão recomendados, uma vez que o seu desempenho depende da interação genótipo-ambiente (Falconer, 1987).

Em experimentos em que são realizados cortes sucessivos e avaliações periódicas de cultivares ao longo do tempo, torna-se possível estimar parâmetros importantes, como a estabilidade (previsibilidade do genótipo em responder à melhoria do ambiente), indispensável para orientar os trabalhos de melhoramento (Cruz \& Regazzi, 1997) e que, no entanto, ainda são raros em

\footnotetext{
1. Projeto financiado pela FENORTE.

2. Aluno de Pós-Graduação do Programa de Produção Vegetal LMGV/CCTA/UENF. Av. Alberto Lamego, 2000. Horto. 28015-620. Campos dos Goytacazes, RJ. rogdaher@uenf.br

3. Professor Associado LMGV/CCTA/UENF.

4. Engenheiro Agrônomo, Dr., Embrapa Gado de Leite, Rua Eugênio do Nascimento, 610, 36038-330, Juiz de Fora, MG.
} 
plantas forrageiras. Vencovsky e Barriga (1992) afirmam que alguns autores preferem utilizar o termo estabilidade para se referir ao comportamento de cultivares ao longo de diferentes anos, num dado local (estabilidade temporal). Essa seria a estabilidade que mais interessa ao produtor. Quando os ambientes são constituídos de diferentes locais, num dado ano, prefere-se usar o termo adaptabilidade (estabilidade geográfica).

Ferreira et al. (2000) avaliaram a estabilidade de cultivares de alfafa em três localidades diferentes: Coronel Pacheco e Sete Lagoas, em Minas Gerais, e Chapecó, em Santa Catarina. Considerando-se cada corte como ambiente, os autores estimaram os parâmetros $\hat{\beta}_{\text {li e }} \hat{\sigma}_{\text {di }}^{2}$ de Eberhart \& Russel (1966) para as estações das águas e da seca e, após agruparem os ambientes em épocas distintas (águas e seca), estimaram os índices de estabilidade $\mathrm{P}_{\mathrm{i}}$ propostos por Carneiro (1998), adaptado de Lin \& Binns (1988) em condição geral, para ambientes considerados favoráveis e para ambientes considerados desfavoráveis.
Conduziu-se este trabalho com o objetivo de obter estimativas de parâmetros de estabilidade da produção forrageira de clones de capim-elefante, num total de 12 cortes realizados no período de dois anos, nas condições edafoclimáticas do norte do Estado do Rio de Janeiro.

\section{MATERIAL E MÉTODOS}

Quatorze novos clones (híbridos intraespecíficos) de capim-elefante (Pennisetum purpureum Schum.), provenientes do Programa de Melhoramento da espécie na Embrapa Gado de Leite, e três cultivares testemunhas (Pioneiro, Mineiro e Taiwan A-146) (Tabela 1) foram avaliados no delineamento em blocos ao acaso, com 4 repetições. A parcela foi composta por 4 linhas de quatro metros, espaçadas de um metro, sendo consideradas bordaduras as duas linhas das extremidades e como área útil da parcela consideraram-se apenas dois metros centrais das duas linhas do interior da parcela, desprezando-se um metro em cada extremidade de cada linha central, totalizando-se quatro metros quadrados.

TABELA 1 - Identificação dos clones (híbridos intraespecíficos) e das cultivares testemunhas de capim-elefante avaliados em Campos dos Goytacazes, RJ.

\begin{tabular}{ccrcc}
\hline $\begin{array}{c}\text { Número de } \\
\text { Identificação } \\
\text { UENF }\end{array}$ & $\begin{array}{c}\text { Identificação da Embrapa } \\
\text { Gado de Leite }\end{array}$ & Progenitor Masculino & Progenitor Feminino \\
\hline 1 & CNPGL 91 F19-1 & Mercker Pinda & X & Elefante da Colômbia \\
2 & CNPGL 91 F10-5 & Taiwan A-148 & X & Mercker Santa Rita \\
3 & CNPGL 91 F27-5 & Mineiro & X & Mercker Santa Rita \\
4 & CNPGL 91 F02-5 & Mercker & X & Mercker Santa Rita \\
5 & CNPGL 91 F01-2 & Elefante da Colômbia & X & Mercker \\
6 & CNPGL 91 F13-2 & Albano & X & Mercker \\
7 & CNPGL 91 F25-3 & Pusa Napier n' 1 & X & Três Rios \\
8 & CNPGL 91 F10-2 & Taiwan A-148 & X & Mercker \\
9 & CNPGL 91 F02-4 & Mercker & X & Napier Volta Grande \\
10 & CNPGL 91 F11-2 & Porto Rico 534-B & X & Mercker \\
11 & CNPGL 91 F34-1 & Duro de Volta Grande & X & Elefante da Colômbia \\
12 & Pioneiro & Mineiro & X & Elefante da Colômbia \\
13 & CNPGL 91 F28-1 & Mole de Volta Grande & X & Elefante da Colômbia \\
14 & CNPGL 91 F06-3 & Pusa Napier nº 2 & X & Três Rios \\
15 & CNPGL 91 F17-5 & Costa Rica & X & Mercker Santa Rita \\
16 & Mineiro & Cultivar testemunha \\
17 & Taiwan A-146 & Cultivar testemunha \\
\hline & & &
\end{tabular}


O experimento foi instalado no Setor de Forragicultura do Laboratório de Zootecnia e Nutrição Animal do Centro de Ciências e Tecnologias Agropecuárias da Universidade Estadual do Norte-Fluminense, em Campos dos Goytacazes, RJ, num solo de terraço classificado como Latossolo Amarelo, distrófico, textura arenosa, conduzido de fevereiro de 1995 a dezembro de 1997 , totalizando 12 cortes (09/10/95, 18/12/95, 15/02/96, 23/04/96, 10/07/96, 23/10/96, 18/12/96, 25/02/97, 28/04/97, 23/07/97, 23/10/97 e 22/12/97). Análises efetuadas antes da instalação do experimento revelaram as seguintes características: pH (em água) 5,0; fósforo disponível (ppm) 11,0; potássio (ppm) 2,0; Ca+Mg (meq/100 $\left.\mathrm{cm}^{3}\right) \quad 4,3 ; \mathrm{Al}$ trocável $\left(\mathrm{meq} / 100 \mathrm{~cm}^{3}\right)$ 0,2 e matéria orgânica (\%) 2,5. Procedeu-se à calagem na dose de 2 t/ha de calcário dolomítico PRNT $90 \%$ (com base em $65 \%$ da saturação de bases). O plantio foi realizado em 17/02/95, por meio de plantas inteiras, dispostas pé com ponta, em fileiras duplas, em sulcos de $10 \mathrm{~cm}$ de profundidade, acompanhado da seguinte adubação: $100 \mathrm{~kg} / \mathrm{ha}$ de $\mathrm{P}_{2} \mathrm{O}_{5}, 30 \mathrm{~kg} / \mathrm{ha}$ de $\mathrm{K}_{2} \mathrm{O}$ e $15 \mathrm{~kg} / \mathrm{ha}$ de $\mathrm{N}$, incorporada no fundo do sulco. Após 50 dias do plantio, complementou-se a adubação com cobertura de $30 \mathrm{~kg} / \mathrm{ha}$ de $\mathrm{K}_{2} \mathrm{O}$ e $25 \mathrm{~kg} / \mathrm{ha}$ de $\mathrm{N}$ e, em 22/06/95, realizou-se o corte de uniformização. Após cada corte, foi efetuada adubação em cobertura com 60 kg/ha de $\mathrm{K}_{2} \mathrm{O}$ e $50 \mathrm{~kg} / \mathrm{ha}$ de $\mathrm{N}$ e uma adubação fosfatada no início da época das águas (100 $\mathrm{kg} / \mathrm{ha}$ de $\mathrm{P}_{2} \mathrm{O}_{5}$ ). Avaliou-se a produção de matéria seca (MS) por corte, em kg/ha/corte, totalizando 12 cortes.

Obtidos os dados, fez-se uma análise de variância individual para cada ambiente para produtividade, com base na média das parcelas. A seguir, procedeu-se à análise de variância conjunta com base no modelo misto:

$$
Y_{i j k}=\mu+G_{i}+A_{j}+G_{i j}+B / A_{j k}+\varepsilon_{i j k},
$$

em que:

$\mathrm{Y}_{\mathrm{ijk}}=$ valor observado do i-ésimo genótipo no k-ésimo bloco dentro do j-ésimo ambiente;

$\mu=$ média geral;

$\mathrm{G}_{\mathrm{i}}=$ efeito fixo do i-ésimo genótipo;

$\mathrm{A}_{\mathrm{j}}=$ efeito aleatório do j-ésimo ambiente;

$\mathrm{GA}_{\mathrm{ij}}=$ efeito da interação do i-ésimo genótipo com o jésimo ambiente;

$\mathrm{B} / \mathrm{A}_{\mathrm{jk}}=$ efeito do k-ésimo bloco dentro do j-ésimo ambiente; e

$\varepsilon_{\mathrm{ijk}}=$ erro experimental.

Para a obtenção das estimativas de estabilidade, foram empregadas metodologias baseadas em análise de variância (Plaisted \& Peterson, 1959) e em análise nãoparamétrica (Lin \& Binns, 1988; Kang, 1988), buscando maior confiabilidade nas estimativas obtidas.
Considerou-se aqui os sucessivos cortes como ambientes de avaliação de genótipos.

\section{Método de Plaisted \& Peterson (1959)}

O parâmetro de estabilidade $\left(\theta_{\mathrm{i}}\right)$ é definido como a média aritmética dos componentes de variância da interação entre pares de genótipos por ambientes $\left(\sigma_{\text {ga }}^{2}\right)$, que envolvem um genótipo particular.

Sua estimativa foi obtida pela expressão:

$$
\theta_{i}=\frac{\sum_{i^{\prime}} \bar{\sigma}_{\text {ga }^{2} i^{\prime}}}{g-1} \text { com } i^{\prime} \neq i
$$

sendo:

$$
\hat{\sigma}_{\text {gaii' }}^{2}=\frac{\frac{S Q\left(G_{i i^{\prime} \times A}\right)}{a-1} Q M R}{r}
$$

em que:

$$
\begin{gathered}
S Q\left(G_{i i}{ }^{\prime} \times A\right)=r / 2\left[d^{2}{ }_{i i^{\prime}}-1 / a\left(Y_{i}-Y_{i^{\prime}}\right)^{2}\right] ; e \\
d^{2}{ }_{i i^{\prime}}=\sum_{j}\left(Y_{i j}-Y_{i^{\prime} j}\right)^{2} \quad(\operatorname{para} j=1,2, \ldots, a)
\end{gathered}
$$

A contribuição relativa de cada genótipo foi calculada por:

$$
\theta_{\mathrm{i}}(\%)=\frac{\theta_{\mathrm{i}} \times 100}{\mathrm{~g} \sigma_{\mathrm{ga}}^{2}}
$$

\section{Método de Lin \& Binns (1988)}

Nesta metodologia, a performance dos acessos foi quantificada pelo índice de estabilidade $\mathrm{P}_{\mathrm{i}}$, que corresponde ao quadrado médio da distância entre a média de um acesso para um dado ambiente e a resposta máxima para o mesmo ambiente, em todos os ambientes avaliados. Dessa forma, o quadrado médio menor indica uma superioridade geral da cultivar em questão, pois quanto menor o valor de $\mathrm{P}_{\mathrm{i}}$, menor será o desvio em torno da produtividade máxima; assim, maior estabilidade está relacionada, obrigatoriamente, com alta produtividade (Lin \& Binns, 1988). rioridade:

A seguinte fórmula define tal medida de supe-

$$
P_{i}=\frac{\sum_{j=1}^{n}\left(X_{i j}-M_{j}\right)^{2}}{2 n}
$$

em que:

$\mathrm{P}_{\mathrm{i}}=$ índice de superioridade do i-ésimo genótipo; 
$\mathrm{X}_{\mathrm{ij}}=$ produtividade do i-ésimo genótipo no j-ésimo local;

$\mathrm{M}_{\mathrm{j}}=$ resposta máxima obtida entre todos os genótipos no j-ésimo local; e

$\mathrm{n}=$ número de locais.

A seguir, procedeu-se à decomposição da expressão para a obtenção do desvio genético e devido à interação.

O desvio genético foi quantificado por:

$$
\frac{\mathrm{n} \overline{(\mathrm{Yi}} \cdot-\overline{\mathrm{M}})^{2}}{2 \mathrm{n}}
$$

O desvio da interação foi obtido pela expressão:

$$
\sum_{j=1}^{n} \frac{\left(Y_{i j}-\bar{Y}_{i}-M_{j}+\bar{M}\right)^{2}}{2 n}
$$

em que:

$$
Y_{i .}=\frac{\sum_{j=1}^{n} Y_{i j}}{n} \text { é a média do genótipo i; e }
$$

$\mathrm{M}=\frac{\sum_{\mathrm{j}=1}^{\mathrm{n}} \mathrm{M}_{\mathrm{j}}}{\mathrm{n}}$ é a média dos genótipos com resposta máxima.

\section{Método de Kang (1988)}

Por esta metodologia, procedeu-se ao ranqueamento dos genótipos, em ordem crescente, com base nos estimadores de $\theta_{\mathrm{i}}$, de Plaisted \& Peterson (1959). A seguir, ranquearam-se os genótipos em ordem decrescente, com base nas estimativas das médias de produção. Os valores dos ranqueamentos de cada genótipo foram, então, somados, obtendo-se a soma das classificações, que se constitui no estimador de Kang (1988).

Por conseguinte, os genótipos com menores valores da soma de 'ranks` foram descritos como os mais estáveis e produtivos.

\section{RESULTADOS E DISCUSSÃO}

Análise de médias, de variâncias individuais e de variância conjunta

A amplitude de variação da produção forrageira entre os clones foi considerável, alcançando valores extremos como o obtido pelo híbrido CNPGL 91 F27-5, com média de 13484,0 kgMS/ha no ambiente 2, e o híbrido CNPGL 91 F19-1, que apresentou o reduzido va- lor de 411,8 kgMS/ha no ambiente 10 (Tabela 2). Com relação à ocorrência de variação nas médias por ambiente, verificou-se oscilação de $8.453,6 \mathrm{kgMS} / \mathrm{ha}$ para o ambiente 2 a 755,4 kgMS/ha para o ambiente 10, e nas médias dos clones (tratamentos), houve variação de 4724,6 kgMS/ha para o híbrido CNPGL 91 F-27-5 a 2745,6 kgMS/ha para o híbrido CNPGL 91 F10-2.

Os valores de quadrados médios de resíduo (QMR) obtidos das análises de variância individuais (por ambiente) de produção de matéria seca (MS), em $\mathrm{kg} / \mathrm{ha} /$ corte (Tabela 3), resultaram numa relação entre o maior e o menor valor de QMR igual a 184,88, o que indica o alto grau de heterogeneidade das variâncias nos ambientes avaliados individualmente, impossibilitando a inclusão de todos os ambientes na análise de variância conjunta. Considerando como aceitável a proporção 7:1 (Cruz \& Regazzi, 1997), procedeu-se ao descarte dos ambientes 1, 2, 5 e 10, obtendo-se, então, a proporção de 4,94 entre o maior e o menor valor de QMR para os oito ambientes restantes, indicando nesses relativa homogeneidade de variâncias, permitindo a utilização desses ambientes na análise variância conjunta (Tabela 4).

As fontes de variação tratamentos, ambientes e a interação dessas apresentaram-se significativas, pelo teste F, aos níveis de 1,1 e $5 \%$ de probabilidade, respectivamente. A significância da interação torna possível o estudo da estabilidade fenotípica dos clones, visando à indicação de genótipos promissores a ambientes particulares.

\section{Estimativas dos Parâmetros de Estabilidade}

\section{Plaisted \& Peterson (1959)}

A avaliação da estabilidade do comportamento dos acessos por meio do método (baseado em análise de variância) de Plaisted \& Peterson (1959) mostrou que os clones mais estáveis, por apresentarem menores valores para a estimativa $\theta_{\mathrm{i}}$ foram, em ordem crescente de magnitude dos sete primeiros clones, CNPGL 91 F17-5 (15), CNPGL 91 F34-1 (11), CNPGL 91 F01-2 (5), CNPGL 91 F13-2 (6), CNPGL 91 F11-2 (10), Pioneiro (12) e Mineiro (16), com valores percentuais de 3,57; 3,$66 ; 3,78 ; 3,79 ; 3,99 ; 4,58$ e 4,67, respectivamente, conforme descrito na Tabela 5.

De acordo com esse método, de um modo geral, não houve concordância entre estabilidade e produtividade, ou seja, os clones mais produtivos não se apresentaram necessariamente como os mais estáveis, conforme pode ser verificado pelo clone CNPGL 91 F27-5 (3), que apresentou a maior média de produtividade de matéria seca (3.510,4 kgMS/corte), tendo, por outro lado, apresentado o segundo maior valor de $\theta_{\mathrm{i}}(662.910,2)$, correspondendo a $13,73 \%$ do total, ou seja, pequena estabilidade. 
TABELA 2 - Produção de matéria seca (em kg/ha/corte) de quatorze híbridos intraespecíficos e três cultivares de capim-elefante avaliados em doze cortes (ambientes) em Campos dos Goytacazes, RJ.

\begin{tabular}{|c|c|c|c|c|c|c|c|c|c|c|c|c|c|}
\hline \multirow{2}{*}{$\begin{array}{l}\text { Ident. } \\
\text { UENF }\end{array}$} & \multicolumn{12}{|c|}{ Ambientes (Cortes) } & \multirow{2}{*}{ Médias } \\
\hline & 1 & 2 & 3 & 4 & 5 & 6 & 7 & 8 & 9 & 10 & 11 & 12 & \\
\hline 1 & 9727.5 & 7313.3 & 2036.8 & 1762.0 & 779.8 & 4601.5 & 3161.8 & 3424.0 & 2603.5 & 411.8 & 821.5 & 1488.0 & 3177.6 \\
\hline 2 & 6185.3 & 9454.3 & 2631.8 & 2893.0 & 933.5 & 4218.3 & 2409.0 & 2697.0 & 3335.5 & 423.5 & 1348.0 & 1606.3 & 3177.9 \\
\hline 3 & 12551.3 & 13484.0 & 5995.0 & 3254.8 & 1402.8 & 4487.3 & 3812.0 & 3644.3 & 3064.5 & 1174.0 & 2085.0 & 1740.8 & 4724.6 \\
\hline 4 & 8279.3 & 7256.5 & 2523.0 & 2399.8 & 1127.3 & 4569.0 & 3504.5 & 3276.0 & 3662.8 & 1041.3 & 1776.8 & 2208.0 & 3468.7 \\
\hline 5 & 5457.5 & 8091.5 & 2818.0 & 2993.5 & 1299.3 & 3353.8 & 2794.0 & 2748.5 & 3004.3 & 590.8 & 1362.3 & 1728.8 & 3020.2 \\
\hline 6 & 6383.8 & 6738.5 & 2305.8 & 2278.0 & 1029.3 & 3160.0 & 3310.5 & 2943.8 & 2661.0 & 742.0 & 1461.3 & 1733.0 & 2895.6 \\
\hline 7 & 8625.8 & 8596.3 & 3007.0 & 3117.8 & 857.0 & 4612.0 & 3356.5 & 3292.0 & 2563.3 & 685.3 & 2331.8 & 2381.8 & 3618.9 \\
\hline 8 & 6641.5 & 6004.8 & 1856.8 & 2391.3 & 872.0 & 3111.3 & 2259.0 & 2743.0 & 3137.5 & 713.5 & 1704.3 & 1512.3 & 2745.6 \\
\hline 9 & 4926.5 & 8662.5 & 2905.3 & 2236.5 & 1329.5 & 4288.0 & 4200.0 & 3745.8 & 3450.8 & 799.3 & 1680.8 & 2390.8 & 3384.6 \\
\hline 10 & 11626.5 & 10166.0 & 3349.3 & 3387.0 & 1269.8 & 4239.0 & 3400.5 & 3652.3 & 3845.5 & 757.5 & 2463.0 & 2010.5 & 4180.6 \\
\hline 11 & 8434.5 & 9422.0 & 3288.0 & 2092.5 & 1081.5 & 5714.3 & 2695.8 & 2721.3 & 2818.8 & 747.8 & 1353.8 & 1695.0 & 3505.4 \\
\hline 12 & 10526.0 & 10738.3 & 3618.8 & 3181.0 & 1580.8 & 3794.3 & 3343.0 & 3270.3 & 3187.5 & 1229.8 & 2885.8 & 2609.5 & 4163.7 \\
\hline 13 & 4983.5 & 5244.5 & 2936.8 & 3183.0 & 1032.5 & 3735.3 & 3216.5 & 2862.3 & 4108.8 & 571.8 & 1910.5 & 2682.5 & 3039.0 \\
\hline 14 & 12003.3 & 9213.3 & 3673.8 & 2296.5 & 1209.3 & 3552.0 & 3168.5 & 3618.8 & 3974.8 & 969.3 & 2607.0 & 1620.5 & 3992.2 \\
\hline 15 & 7032.5 & 8776.8 & 3089.5 & 2458.0 & 1042.3 & 3517.8 & 3434.3 & 2828.0 & 2787.3 & 698.8 & 1364.8 & 2301.0 & 3277.6 \\
\hline 16 & 6365.3 & 6033.3 & 3158.8 & 2667.5 & 1288.3 & 3213.0 & 3544.0 & 2565.8 & 2310.5 & 732.8 & 1375.3 & 1872.5 & 2927.2 \\
\hline 17 & 10071.3 & 8515.5 & 3145.8 & 1980.5 & 947.5 & 3643.0 & 3134.5 & 2558.8 & 2995.8 & 552.8 & 1190.5 & 4625.3 & 3613.4 \\
\hline Médias & 8224.8 & 8453.6 & 3078.8 & 2621.9 & 1122.5 & 3988.8 & 3220.3 & 3093.6 & 3147.8 & 755.4 & 1748.4 & 2129.8 & 3465.4 \\
\hline
\end{tabular}

TABELA 3 - Resultado das análises de variância individuais de produção de matéria seca (em kg/ha/corte) para 12 ambientes (cortes) avaliados nas condições de Campos dos Goytacazes, RJ.

\begin{tabular}{ccccc}
\hline Ambiente & QMBloco & QMTratamento & QMResíduo & F \\
\hline 1 & 197786734.19 & 24928863.63 & 16169336.15 & 1.54 \\
2 & 47487929.45 & 16026172.65 & 7934976.24 & 2.01 \\
3 & 1344627.42 & 3271687.07 & 1411769.93 & 2.31 \\
4 & 3657797.50 & 1001504.15 & 597193.50 & 1.67 \\
5 & 376465.41 & 190326.77 & 110968.54 & 1.71 \\
6 & 881205.12 & 1220609.51 & 1029075.62 & 1.19 \\
7 & 4605005.19 & 917034.46 & 597995.74 & 1.53 \\
8 & 7647889.58 & 694637.72 & 374735.28 & 1.85 \\
9 & 1294058.33 & 1087837.28 & 633960.24 & 1.72 \\
10 & 763435.19 & 217599.25 & 87455.75 & 2.48 \\
11 & 707571.52 & 1258268.68 & 494308.49 & 2.54 \\
12 & 2918849.54 & 2248940.71 & 1852968.20 & 1.21 \\
\hline G.L. & 3 & 16 & 48 & \\
\hline
\end{tabular}

Método de Lin \& Binns (1988)

Ciênc. agrotec., Lavras. V.27, n.4, p.788-797, jul./ago., 2003 
As estimativas de média de produção de matéria seca (em kg/ha), do parâmetro de estabilidade (baseado em análise não paramétrica) $\left(\mathrm{P}_{\mathrm{i}}\right)$ de Lin \& Binns (1988), bem como a decomposição do parâmetro $\mathrm{P}_{\mathrm{i}}$ em suas partes por causa do desvio genético e da interação, além da contribuição para a interação de quatorze híbridos intraespecíficos e três cultivares de capim-elefante avaliados em oito cortes são apresentadas na Tabela 6.

TABELA 4 - Resumo da análise de variância conjunta da produção de matéria seca (kg/ha) de quatorze clones e três cultivares de capim-elefante em oito ambientes (cortes) em Campos dos Goytacazes, RJ.

\begin{tabular}{lcccc}
\hline \multicolumn{1}{c}{ F.V. } & G.L. & S.Q. & Q.M. & F \\
\hline Bloco/Amb. & 24 & 69171012.705883 & 2882125.529412 & \\
Tratamentos & 16 & 60026883.382353 & 3751680.211397 & $3.303849^{* *}$ \\
Ambientes & 7 & 211102925.411764 & 30157560.773109 & $10.463653^{* *}$ \\
Trat x Amb & 112 & 127181430.588236 & 1135548.487395 & $1.299253^{*}$ \\
Resíduo & 384 & 335616337.794117 & 874000.879672 & \\
\hline Total & 543 & 803098589.882353 & & \\
\hline
\end{tabular}

** Significativo no nível de $1 \%$ de probabilidade;

* Significativo no nível de $5 \%$ de probabilidade.

TABELA 5 - Estimativas de parâmetros de estabilidade propostos por Plaisted \& Peterson (1959) de quatorze híbridos intraespecíficos e três cultivares de capim-elefante avaliados em oito cortes (ambientes) em Campos dos Goytacazes, RJ.

\begin{tabular}{ccccc}
\hline Ident. & \multirow{2}{*}{ Clones } & Média & \multicolumn{2}{c}{ Plaisted \& Peterson (1959) } \\
\cline { 4 - 5 } UENF & & $2.722,5$ & $\theta_{\mathbf{i}}$ & $\theta_{\mathbf{i}}(\boldsymbol{\%})$ \\
\hline 15 & CNPGL 91 F17-5 & $2.484,9$ & $172.525,7$ & 3,57 \\
11 & CNPGL 91 F34-1 & $2.600,3$ & $182.548,7$ & 3,66 \\
5 & CNPGL 91 F01-2 & $2.481,6$ & $183.017,5$ & 3,78 \\
6 & CNPGL 91 F13-2 & $3.293,3$ & $192.933,1$ & 3,79 \\
10 & CNPGL 91 F11-2 & $3.236,2$ & $221.411,6$ & 3,99 \\
12 & Pioneiro & $2.588,4$ & $225.593,9$ & 4,58 \\
16 & Mineiro & $2.989,9$ & $228.378,7$ & 4,67 \\
4 & CNPGL 91 F02-5 & $3.082,7$ & $236.932,5$ & 4,73 \\
7 & CNPGL 91 F25-3 & $3.079,3$ & $238.352,6$ & 4,90 \\
13 & CNPGL 91 F28-1 & $2.642,3$ & $242.143,2$ & 4,93 \\
2 & CNPGL 91 F10-5 & $2.339,4$ & $243.656,5$ & 5,01 \\
8 & CNPGL 91 F10-2 & $3.112,2$ & $249.688,2$ & 5,04 \\
9 & CNPGL 91 F02-4 & $3.063,9$ & $306.706,1$ & 5,17 \\
14 & CNPGL 91 F06-3 & $2.487,3$ & $367.332,2$ & 6,35 \\
1 & CNPGL 91 F19-1 & $3.510,4$ & $662.910,2$ & 7,61 \\
3 & CNPGL 91 F27-5 & $2.909,2$ & $695.299,3$ & 13,73 \\
17 & Taiwan A-146 & $2.860,2$ & $283.887,1$ & 14,40 \\
\hline
\end{tabular}


As estimativas do parâmetro $\mathrm{P}_{\mathrm{i}}$ variaram amplamente, desde $640.303,4$ para o clone 3 (CNPGL 91 F275) até 2.323.183,6 para o clone 8 (CNPGL 91 F10-2). De um modo geral, houve um relacionamento inverso entre o parâmetro de estabilidade $\mathrm{P}_{\mathrm{i}} \mathrm{e}$ as médias de produtividade dos clones, indicando a aplicabilidade dessas estimativas de estabilidade para a avaliação de genótipos de ciclo perene submetidos a cortes sucessivos. A capacidade desse parâmetro em detectar o comportamento genotípico dos clones se baseia na utilização de desvios entre o genótipo avaliado e a produtividade máxima dentro de cada ambiente. Assim, baixos valores de $\mathrm{P}_{\mathrm{i}}$ para determinado genótipo evidenciam que o mesmo esteve próximo do máximo nos cortes realizados.

Os sete primeiros genótipos que se destacaram com os menores valores de $\mathrm{P}_{\mathrm{i}}$ foram, em ordem crescente de $\mathrm{P}_{\mathrm{i}}$ : 3 (CNPGL 91 F27-5), 12 (cultivar Pioneiro), 10 (CNPGL 91 F11-2), 13 (CNPGL 91 F281), 7 (CNPGL 91 F25-3), 17 (cultivar Taiwan A-146) e 9 (CNPGL 91 F02-4), com respectivos valores de $\mathrm{Pi}$ de $640.303,4 ; 764.330,8 ; 929.500,0 ; 1039.777,1$; $1102.828,4 ; 1105.701,1$ e $1115.781,4$.

Considerando-se os componentes das estimativas de $\mathrm{P}_{\mathrm{i}}$ que são atribuídos ao desvio genético e à interação genótipos por ambientes (Tabela 6), pode-se observar que em todos os genótipos avaliados o componente genético foi superior ao componente devido à interação. No entanto, retornando aos sete genótipos citados como de menores valores de $\mathrm{P}_{\mathrm{i}}$, pode-se observar que, para os clones 3 (CNPGL 91 F27-5) e 10 (CNPGL 91 F11-2), a porcentagem para o valor genético foi ligeiramente inferior em relação aos demais $(52,8 \%$ e 58,1 , respectivamente). Considerando que o genótipo ideal deve apresentar o menor valor possível de $\mathrm{P}_{\mathrm{i}}$ e maior influência do componente genético, pode-se, por conseguinte, verificar que os clones de maior interesse foram 12 (cultivar Pioneiro), 13 (CNPGL 91 F28-1), 7 (CNPGL 91 F25-3), 17 (cultivar Taiwan A-146) e 9 (CNPGL 91 F02-4).

A decomposição do parâmetro de estabilidade proposto por Lin \& Binns (1988) em partes devidas a ambientes favoráveis (aqueles com média superior à média geral) e a ambientes desfavoráveis (cujas médias são inferiores à média geral), apresentada na tabela 6 , possibilita conhecer a resposta dos genótipos às alterações das condições do ambiente. Observando os sete primeiros genótipos com menor valor de $\mathrm{P}_{\mathrm{i}}$ geral, e menor $\mathrm{Pi}$ tanto em ambientes favoráveis quanto em ambientes desfavoráveis (Tabela 6), verifica-se a presença constante desses genótipos, ocorrendo apenas alteração na posição que os mesmos ocupam em cada situação. Nos ambientes favoráveis, destacou-se como muito promissor o clone 3 (CNPGL 91 F27-5), com Pi igual a 126.582,3. Em seguida, ainda em ambientes favoráveis, também mostraram-se promissores os clones 14 (CNPGL 91 F06-3) e 10 (CNPGL 91 F11-2), com valores de Pi de 760.974,5 e 785.636,5. Considerando apenas os ambientes desfavoráveis, destacaram-se como responsivos os clones 13 (CNPGL 91 F28-1), 7 (CNPGL 91 F25-3) e 17 (cultivar Taiwan A-146). Os clones 12 (cultivar Pioneiro) e 9 (CNPGL 91 F02-4) mostraram alta estabilidade, tanto em ambientes favoráveis quanto em ambientes desfavoráveis (Tabela 6).

\section{Método de Kang (1988)}

A baixa aplicabilidade da análise isolada das estimativas de estabilidade $\theta_{\mathrm{i}}$ (Plaisted \& Peterson, 1959) encontradas no presente trabalho (tabela 5) propiciou o emprego da metodologia de Kang (1988), a qual se utiliza de um somatório de classificações para avaliar a performance genotípica. De acordo com essa metodologia, os genótipos são ordenados em duas colunas: uma referente ao valor do parâmetro de estabilidade $\left(\theta_{\mathrm{i}}\right)$, e outra referente à média de produtividade. Para cada genótipo, é feito um ranqueamento do seu correspondente valor de $\theta_{\mathrm{i}}$ e da sua média; o primeiro em ordem crescente e o segundo em ordem decrescente. Os genótipos que apresentarem menor soma das classificações serão os mais indicados, visto que serão os mais estáveis e mais produtivos.

Os resultados obtidos de estimativas do parâmetro de estabilidade de Kang (1988), encontrados na Tabela 8 permitiram ratificar o bom desempenho dos clones 10 (CNPGL 91 F11-2), 12 (cultivar Pioneiro), 7 (CNPGL 91 F25-3) e 13 (CNPGL 91 F28-1) e incluíram os clones 15 (CNPGL 91 F17-5), 5 (CNPGL 91 F01-2) e 4 (CNPGL 91 F02-5) no rol dos sete primeiros de maior estabilidade.

O clone 3 (CNPGL 91 F27-5), destacado como muito promissor pelo método de Lin \& Binns (1988), ocupou a oitava posição do ranqueamento de Kang (1988). Outro excluído foi o nono colocado no ranqueamento de Kang (1988), o clone 9 (CNPGL 91 F02-4), que mostrou alta estabilidade tanto em ambientes favoráveis quanto em ambientes desfavoráveis (Tabela 7).

A análise comparativa envolvendo os sete primeiros clones melhores colocados em cada uma das metodologias utilizadas (Plaisted \& Peterson, 1959; Lin \& Binns, 1988; Kang, 1988) revelou que apenas os clones CNPGL 91 F11-2 e a cultivar Pioneiro permaneceram consistentemente entre os sete primeiros,

Ciênc. agrotec., Lavras. V.27, n.4, p.788-797, jul./ago., 2003 
revelando-se materiais superiores em produtividade e para as condições do município de Campos dos Goyestabilidade, podendo-se considerá-los promissores tacazes, RJ.

TABELA 6 - Estimativas de parâmetros de estabilidade (Pi) propostos por Lin \& Binns (1988), desvios genético e da interação e decomposição em partes devidas a ambientes favoráveis e desfavoráveis de quatorze híbridos intraespecíficos e três cultivares de capim-elefante avaliados em oito cortes (ambientes) em Campos dos Goytacazes, RJ.

\begin{tabular}{|c|c|c|c|c|c|c|c|c|}
\hline \multicolumn{3}{|c|}{$\begin{array}{c}\text { Estimativas de Parâmetros } \\
\text { de Estabilidade }\end{array}$} & \multirow{2}{*}{\multicolumn{2}{|c|}{ Desvios }} & \multirow{3}{*}{$\begin{array}{l}\text { Perc. para } \\
\text { Desvio } \\
\text { Genético }\end{array}$} & \multirow{3}{*}{$\begin{array}{c}\text { Contrib. } \\
\text { para a In- } \\
\text { teração }(\%)\end{array}$} & \multirow{2}{*}{\multicolumn{2}{|c|}{$\mathbf{P i}$}} \\
\hline \multicolumn{3}{|c|}{ Geral } & & & & & & \\
\hline $\begin{array}{l}\text { Ident. } \\
\text { UENF }\end{array}$ & Médias & $\mathbf{P i}$ & Genético & Interação & & & $\begin{array}{l}\text { Ambientes } \\
\text { favoráveis }\end{array}$ & $\begin{array}{c}\text { Ambientes } \\
\text { desfavoráveis }\end{array}$ \\
\hline 3 & $3.510,4$ & $640.303,4$ & 338.074 & 302.229 & 52,8 & 6,74 & $126.582,3$ & $1496.505,1$ \\
\hline 12 & $3.236,3$ & $764.330,8$ & 601.123 & 163.208 & 78,6 & 3,64 & $812.360,9$ & $684.280,7$ \\
\hline 10 & $3.293,3$ & $929.500,0$ & 540.119 & 389.381 & 58,1 & 8,68 & $785.636,5$ & $1169.272,5$ \\
\hline 13 & $3.079,4$ & $1039.777,1$ & 785.359 & 254.419 & 75,5 & 5,67 & $1186.942,8$ & $794.501,0$ \\
\hline 7 & $3.082,8$ & $1102.828,4$ & 781.213 & 321.616 & 70,8 & 7,17 & $1223.255,0$ & $902.117,3$ \\
\hline 17 & $2.909,3$ & $1105.701,1$ & 1013.133 & 92.568 & 91,6 & 2,06 & $1283.910,2$ & $808.685,8$ \\
\hline 9 & $3.112,2$ & $1115.781,4$ & 744.812 & 370.970 & 66,8 & 0,83 & $1008.383,7$ & $1294.777,6$ \\
\hline 14 & $3.064,0$ & $1119.072,5$ & 804.865 & 314.208 & 71,9 & 7,01 & $760.974,5$ & $1715.902,4$ \\
\hline 4 & $2.990,0$ & $1312.945,6$ & 901.490 & 411.455 & 68,7 & 9,18 & $1295.948,9$ & $1341.273,5$ \\
\hline 15 & $2.722,6$ & $1336.975,8$ & 1296.304 & 40.672 & 97,0 & 0,91 & $1281.299,2$ & $1429.770,0$ \\
\hline 16 & $2.588,4$ & $1589.567,4$ & 1521.315 & 68.252 & 95,7 & 1,52 & $1505.615,6$ & $1729.487,2$ \\
\hline 5 & $2.600,3$ & $1670.837,0$ & 1500.510 & 170.327 & 89,8 & 3,80 & $1586.778,6$ & $1810.934,5$ \\
\hline 11 & $2.484,9$ & $1679.115,7$ & 1178.594 & 500.522 & 70,2 & 11,16 & $1425.673,3$ & $2101.519,9$ \\
\hline 2 & $2.642,3$ & $1755.891,4$ & 1428.686 & 327.205 & 81,4 & 7,30 & $1637.119,0$ & $1953.845,3$ \\
\hline 6 & $2.481,7$ & $1929.500,5$ & 1713.219 & 216.282 & 88,8 & 4,82 & $1924.781,6$ & $1937.365,2$ \\
\hline 1 & $2.487,4$ & $2241.106,4$ & 1702.649 & 538.457 & 76,0 & 12,01 & $1911.361,2$ & $2790.681,8$ \\
\hline 8 & $2.339,4$ & $2323.183,6$ & 1986.650 & 336.534 & 85,5 & 7,50 & $2509.270,8$ & $2013.038,2$ \\
\hline
\end{tabular}


TABELA 7 - Estimativas do parâmetro de estabilidade de Kang (1988), com base no ranqueamento de $\theta_{\mathrm{i}}$, de Plaisted \& Peterson (1959) e das médias de produtividade

\begin{tabular}{cccccc}
\hline $\begin{array}{c}\text { Identificação } \\
\text { UENF }\end{array}$ & $\begin{array}{c}\text { Estimativa de } \theta_{\mathbf{i}}(\boldsymbol{\%}) \\
\text { Plaisted \& Peterson } \\
(\mathbf{1 9 5 9 )}\end{array}$ & $\begin{array}{c}\text { Classificação } \\
\text { de } \theta_{\mathbf{i}}\end{array}$ & $\begin{array}{c}\text { Médias de } \\
\text { produtividade }\end{array}$ & $\begin{array}{c}\text { Classificação } \\
\text { das médias de } \\
\text { produtividade }\end{array}$ & $\begin{array}{c}\text { Soma das } \\
\text { classificaçóes } \\
\text { Kang (1988) }\end{array}$ \\
\hline 10 & 3.99 & 5 & 3293.3 & 2 & 7 \\
12 & 4.58 & 6 & 3236.2 & 3 & 9 \\
15 & 3.57 & 1 & 2722.5 & 10 & 11 \\
7 & 4.90 & 9 & 3082.7 & 5 & 14 \\
5 & 3.78 & 3 & 2600.3 & 12 & 16 \\
13 & 4.93 & 10 & 3079.3 & 6 & 16 \\
4 & 4.73 & 16 & 2989.9 & 8 & 17 \\
3 & 13.73 & 13 & 3510.4 & 1 & 17 \\
9 & 5.17 & 2 & 3112.2 & 4 & 20 \\
11 & 3.66 & 7 & 2484.9 & 15 & 20 \\
16 & 4.67 & 4 & 2588.4 & 13 & 21 \\
6 & 3.79 & 2481.6 & 16 & 22 \\
14 & 6.35 & 14 & 3063.9 & 7 & 26 \\
1 & 5.01 & 11 & 2642.3 & 11 & 29 \\
\hline
\end{tabular}

\section{CONCLUSÕES}

O clone CNPGL 91 F11-2 e a cultivar Pioneiro revelaram-se materiais superiores em produtividade e estabilidade em relação aos demais, podendo ser considerados promissores para as condições do município de Campos dos Goytacazes, RJ. Entretanto, com o intuito de se ratificar essa recomendação, sugere-se repetir a avaliação por mais dois anos, já que existem materiais, como o clone CNPGL 91 F27-5, com elevados valores de produtividade, entre outros.

\section{REFERÊNCIAS BIBLIOGRÁFICAS}

CARNEIRO, P. C. S. Novas metodologias de análise da adaptabilidade e estabilidade de comportamento. 1998. $93 \mathrm{f}$. Thesis (Doctoral in Genetics and Improvement of Plants) - Universidade Federal de Viçosa, Viçosa.

CRUZ, C. D.; REGAZZI, A. J. Modelos biométricos aplicados ao melhoramento genético. Viçosa: UFV, 1997. $390 \mathrm{p}$.
DAHER, R. F.; MOZZER, O. L.; GONÇALVES, T. M. Comparação de 32 cultivares e híbridos de capim-elefante (Pennisetum purpureum Schum.). In: SIMPÓSIO SOBRE CAPIM-ELEFANTE, 1990, Juiz de Fora. Anais... Juiz de Fora: EMBRAPA-CNPGL, 1990. p. 183.

EBERHART, S. A.; RUSSEL, W. A. Stability parameters for comparing varieties. Crop Science, Madison, v. 36, p. 36-40, 1966.

EMPRESA BRASILEIRA DE PESQUISA AGROPECUÁRIA. Capim-elefante: curso de pecuária leiteira. 2. ed. Coronel Pacheco, 1993. 34 p. (Documentos, 43).

FALCONER, D. S. Introdução à genética quantitativa. Viçosa: UFV, 1987. 279 p.

FERREIRA, R. P.; BOTREL, M. A.; CRUZ, C. D.; MIRANDA, M.; ROCHA, R.; VIANA, M. C. N.; ASSIS, G. M. L.; FERNANDES, E. N. Adaptabilidade e estabilidade em cultivares de alfafa. Ciência e Agrotecnologia, Lavras, v. 24, n. 3, p. 743-755, 2000. 
KANG, M. J. A rank-sum method for selecting highyelding, stable corn genotypes. Cereal Research Communities, Szeged, v. 16, p. 113-115, 1988.

LIN, C. S.; BINNS, M. R. A superiority measure of cultivar performance for cultivar $\mathrm{x}$ location data. Canadian Journal of Plant Science, Ottawa, v. 68, p. 193198, 1988.

MOZZER, O. L. Comparação entre cultivares de capimelefante nas fazendas da Zona da Mata de Minas Gerais. Relatório técnico do Centro Nacional de Pesquisa de Gado de Leite 1981-1985. Coronel Pacheco: EMBRAPA-CNPGL, 1986. p. 88.
PLAISTED, R. L.; PETERSON, L. C. A technique for evaluating the ability of selections to yield consistently in different locations or seasons. American Potato Journal, Orono, v. 36, p. 381-385, 1959.

VENCOVSKY, R.; BARRIGA, P. Genética biométrica no fitomelhoramento. Ribeirão Preto: [s.n.], 1992. $496 \mathrm{p}$. 